\title{
Anti-oxidative and neuroprotective effects of flaxseed on experimental unilateral spinal cord injury in rat
}

\author{
Morteza Gholaminejhad, ${ }^{a}$ Somayeh Arabzadeh, ${ }^{\text {b }}$ Mohammad Akbari, ${ }^{\text {Y }}$ ousef Mohamadi, \\ Gholamreza Hassanzadeh, a*
}

\author{
aDepartment of Anatomy, School of Medicine, Tehran University of Medical Sciences, Tehran, Iran. \\ bDepartment of Animal Physiology, Developmental Biology Laboratory, School of Biology, College of Science, University of Tehran, Tehran, Iran. \\ Correspondence to Gholamreza Hassanzadeh (email: hassanzadeh@tums.ac.ir) \\ (Submitted: 17 April 2017 - Revised version received: 12 May 2017 - Accepted: 28 May 2017 - Published online: 26 June 2017)
}

\begin{abstract}
Objectives Investigation of anti-oxidative and neuroprotective effects of flaxseed on rats with unilateral SCI.
Methods 30 male Wistar rats were randomly assigned to five groups: control (Ctrl), laminectomy, flaxseed, spinal cord injured (SCI) and SCI + flaxseed (treatment) groups. SCl model was induced by placing a $50 \mathrm{~g}$ weight for $5 \mathrm{~min}$ on to a $2.2 \mathrm{~mm} \times 2.5 \mathrm{~mm}$ platform applied to the dura at vertebral level T10. After 4 weeks, the blood of rats in different groups was collected and the influence of flaxseed on antioxidant enzymes and oxidative stress marker level, histologic alterations and locomotion score were assessed.

Results Our results showed that in the SCI group, the mean level of Superoxide dismutase (SOD), glutathione peroxidase (GPX) and catalase (CAT) were significantly decreased $(P<0.01)$, whereas the mean malondialdehyde (MDA) content was significantly increased $(P<0.001)$ in comparison to the laminectomy group. Furthermore, the mean levels of SOD, GPx and CAT in the treatment group was higher than the $\mathrm{SCl}$ group $(P<0.001)$, while, the mean MDA content in the treatment group was significantly less than the $\mathrm{SCl}$ group $(P<0.001)$. In addition, comparison between $\mathrm{SCl}$ and treatment groups determined a significant decrease in tissue degeneration and volume of cavities in the treatment group. However, Basso, Beattie and Bresnahan (BBB) scores were significantly increased in flaxseed-treated rats on days $14(P<0.05), 21$ and $28(P<0.01)$ after surgery compared with the $S C l$ group.

Conclusions Our study for the first time showed the anti-oxidative and neuroprotective effects of flaxseed on experimental unilateral spinal cord injury in rat.

Keywords spinal cord injury, flaxseed, oxidative stress, neuroprotective, anti-oxidative
\end{abstract}

\section{Introduction}

Spinal cord injury (SCI) is a serious health problem which is followed by great psychological disruption and financial burden on patients' families. ${ }^{1}$ The SCI includes two pathological phases that lead finally to the loss of neurons that can have bad consequences. ${ }^{2,3}$ In the primary phase a direct mechanical trauma on the spine causes tearing in tissue and then during secondary phase a cascade of fatal molecular events such as oxidative stress, excitotoxicity and inflammation induces a wide cell death in the injured site. ${ }^{4}$ Another important factors accelerate post-traumatic degeneration due to secondary destruction are oxidative stress and apoptosis. ${ }^{5}$ Disruption of the balance between the production of oxidant compounds and antioxidant defense system induces oxidative stress. Superoxide dismutase (SOD), glutathione peroxidase (GPx) and catalase (CAT) are antioxidant enzymes, which are important in the regulation of oxidant/antioxidant balance. ${ }^{6-8}$ In addition, during lipid peroxidation several products such as malondialdehyde (MDA) and nitric oxide (NO) are generated, which can provide valuable information related to the severity of cell damage caused by free radicals. The changes in the activities of antioxidant enzymes in biological systems reflect the level of oxidative stress. ${ }^{6}$ Although the precise molecular pathway of secondary injury is still controversial, therapeutic strategies that inhibit oxidative stress may lead to SCI improvement.

Flaxseed (Linum usitatissimum) is a seed from flax plant, an annual herb, which is a member of the Linaceae family. ${ }^{9}$ It is a rich source of omega-3 fatty acid a-linolenic acid, lignan phytoestrogen, and soluble fibers. ${ }^{10,11}$ Flaxseed has a remarkable antioxidant effect ${ }^{12}$ and anti-inflammatory effect due to high levels of free radical scavenger properties and Omega-3 fatty acids, ${ }^{13}$ respectively. Furthermore, vitamin E, a fat-soluble vitamin, is present in flaxseed. Vitamin $\mathrm{E}$ has anti-carcinogenic and anti-oxidative effect with the protection of cell constituents from the damaging effects of free radicals that might contribute to cancer development. ${ }^{14}$ Since flaxseed has introduced as an antioxidant herb, the aim of this study was to investigate the anti-oxidative and neuroprotective effects of flaxseed in unilateral SCI model.

\section{Materials and Methods}

\section{Animal Preparations}

Male adult Wistar rats weighing between 250 and 300 g were used in this study $(n=30)$. Animals were kept under constant laboratory conditions, room temperature $\left(20-22^{\circ} \mathrm{C}\right)$, a 12 -h light-dark cycle, and they had free access to food and water. Animal handling was performed following the guidelines of the Iranian Animal Ethics Committee, Tehran University of Medical Sciences rules. Rats were randomly divided into five groups. Each group consists of 6 rats with following description:

1) Control (Crtl) group: without any manipulation.

2) Laminectomy group: with laminectomy without SCI. 
3) Flaxseed group: without any surgery and feed by $10 \%$ flaxseed for 4 weeks.

4) SCI group: with SCI without any treatment.

5) SCI + Flaxseed (Treatment) group: with SCI and feed by $10 \%$ flaxseed for four weeks.

\section{Spinal Cord Injury}

The surgical manner was performed under general anaesthesia induced by intraperitoneal ketamine $(80 \mathrm{mg} / \mathrm{kg})$ (Sigma, Taufkrichen, Germany) and xylazine $(15 \mathrm{mg} / \mathrm{kg})$ (Sigma, Taufkrichen, Germany). Following T8-T12 midline skin incision, paravertebral muscles were dissected, and laminectomy was performed at T10 vertebrae as the Dura mater was to remain intact. The spinal cord was unilaterally pressed by a $50 \mathrm{~g}$ weight for $5 \mathrm{~min}$ using a rectangular plate, which was longitudinally oriented over the spinal cord; the plate had an area of $2.2 \mathrm{~mm} \times 2.5 \mathrm{~mm}$. Rats' body temperatures during the surgery were kept constant between 36 and $37^{\circ} \mathrm{C}$ during surgery. Then, paravertebral fascia and skin were sutured separately.

Upon awakening, rats were neurologically appraised. Food, water and urine uptake were monitored. After 4 weeks, the rats were anaesthetized according to mentioned protocol to collect their blood for further analysis. In this study, we tried to use the minimum number of animals and reduce their suffering.

\section{The Basso, Beattie and Bresnahan locomotion Rating Scale Scores}

The Basso, Beattie and Bresnahan (BBB) were used for the assessment of motor function of rats in different groups. ${ }^{15}$ Two observers who were blind to the treatment group evaluated outcomes. In brief, the BBB scores range from 0 to 21 points, which indicates complete paralysis to normal motor function. The assessments were performed for $24 \mathrm{~h}$ and 7, 14, 21, 28 days after surgery. Rats with a score more than 3 were excluded from the study and with the score less than one selected for the following analysis for $24 \mathrm{~h}$ post-SCI.

\section{Drug Administration}

The flaxseed and normal food were grounded and with a little distilled water mixed. Finally, the plates were prepared by adding $10 \%$ flaxseed to the normal food.

\section{Evaluation of Stress Oxidative Activity}

On week 4 postoperatively, the blood was obtained from the each group. Centrifuged at 1500 r.p.m. for $10 \mathrm{~min}$. Supernatant solution was collected to determine the following biochemical indices. Superoxide dismutase (SOD) (cat \# ZB-SOD48), glutathione peroxidase (GPx) (cat \# ZB-GPX48), catalase (CAT) (cat \# ZB-CAT48) and malondialdehyde (MDA) (cat \# ZB-MDA48) testing kits (Zellbio, Deutschland, Germany) were used to detect the oxidative stress of the injured spinal cord according to their manufacture protocols.

\section{Hematoxylin-Eosin Staining}

For histological analysis, animals were anaesthetized by intraperitoneal ketamine $(80 \mathrm{mg} / \mathrm{kg})$ and xylazine $(15 \mathrm{mg} / \mathrm{kg})$ and perfused with normal saline, 4 weeks after SCI. Then, they were received transcardial perfusion of $0.01 \mathrm{M}$ phosphate-buffered saline (PBS) followed by
$4 \%$ paraformaldehyde $(400 \mathrm{ml} / \mathrm{rat})$ for fixation. After perfusion, a $2-\mathrm{cm}$-long piece of the spinal cord was dissected out from each rat and fixed for $48 \mathrm{~h}$ with mentioned fixation buffer. A 3-mm-thick transverse section was made in the epicentre and $3 \mathrm{~mm}$ cranially and caudally. Afterwards, samples in each group were embedded in paraffin for transverse paraffin sections. The paraffin sections ( $5 \mu \mathrm{m}$ thick) mounted on poly-L-lysine-coated slides for histopathological examination by hematoxylin and eosin staining. In the end, slides were observed under light microscopy (Olympus, Tokyo, Japan).

\section{Statistical Analysis}

Data were analyzed using the statistical software package SPSS.20 statistical software (SPSS, Inc., Chicago, IL, USA). The normality of nominal variables was analyzed by using the Kolmogorov-Smirnov test. For comparing data among groups, one-way ANOVA tests were used. The results were expressed as mean \pm SD. $P$-values $<0.05$ were considered to indicate statistical significance.

\section{Result}

\section{Flaxseed Attenuated Oxidative Stress in Rats Following $\mathrm{SCl}$}

The mean level of SOD, GPx, CAT and the contents of MDA were measured in each group to verify the antioxidant effects of flaxseed treatment 4 weeks after injury. In the SCI group, the mean level of SOD, GPx and CAT were significantly decreased $(P<0.01)$, whereas the mean MDA content was significantly increased $(P<0.001)$ in comparison to the laminectomy group, at 4 weeks after injury (Fig. 1).

The treatment group had a 1.5 fold higher mean level of SOD, GPx and CAT compared with the SCI group $(P<0.001)$, However, SOD and GPx activity in the treatment group were significantly higher than the laminectomy group $(P<0.001)$ (Fig. 1A-C).
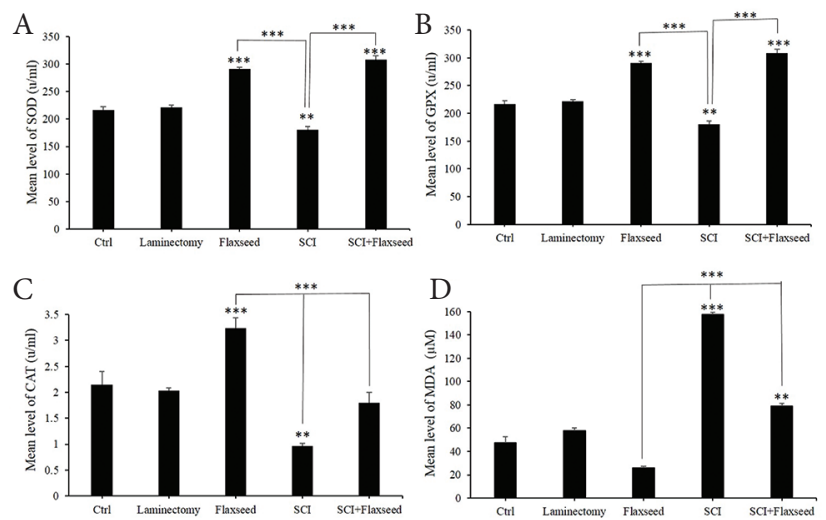

Fig. 1 Flaxseed attenuated oxidative stress in rats following $\mathrm{SCl}$. The mean level of SOD (A), GPX (B) and CAT (C) decreased in the SCI group in comparison with a laminectomy group $(P<0.01)$. However, in the treatment group, SOD (A), GPx (B) and CAT (C) levels significantly increased compared with the $\mathrm{SCl}$ group $(P<0.001)$. The mean MDA (D) content in the $\mathrm{SCl}$ group significantly increased in comparison with laminectomy group $(P<0.001)$. The MDA content in the treatment group was significantly less than the $\mathrm{SCl}$ group $(P<0.001)$, but it was still higher than the laminectomy group $(P<0.01)$. Data were presented as mean + SD. Ctrl: Control. ${ }^{*} P<0.05 ;{ }^{* * P}<0.001 ;{ }^{* *} P<0.001$. 
In addition, the mean MDA content in the treatment group was significantly less than the SCI group $(P<0.001)$, but it was still higher than the laminectomy group $(P<0.01)$ (Fig. 1D). It showed that flaxseed treatment couldn't restore the effect of SCI on MDA content to the control level.

Moreover, the SOD, GPx and CAT level in the flaxseed group were significantly higher than the laminectomy group $(P<0.001)$ (Fig. 1A-C).

\section{Flaxseed Reduced Histological Damage After SCI}

Hematoxylin and eosin staining of spinal cord tissue 4 weeks after SCI showed differences between the control, SCI and treatment groups. Progressive destruction of the dorsal white matter and central grey matter was obvious in the SCI group in comparison to the control group. In the SCI group, following injury, bleeding and recruitment of polymorphonuclear cells, induced cavity in damage location was completely obvious. However, in the treatment group density of polymorphonuclear cells decreased and bleeding wasn't seen. Comparison between SCI and treatment groups determined significant beneficial effects of flaxseed as decreased the volume of contusion-induced cavities and improved tissue structure in the treatment group (Fig. 2).

\section{Flaxseed Improved locomotor Functional Recovery of Rats Following SCl}

The effects of flaxseed on locomotor functional recovery in rats after SCI, were measured by BBB test on days $1,3,7,14,21$ and 28 post SCI. As shown in Figure 3, there isn't any significant difference in BBB scores between the SCI and the treatment group on days 3 and 7 after SCI. However, BBB scores were significantly increased in flaxseed-treated rats on days 14 $(P<0.05), 21$ and $28(P<0.01)$ after surgery compared with the SCI group.
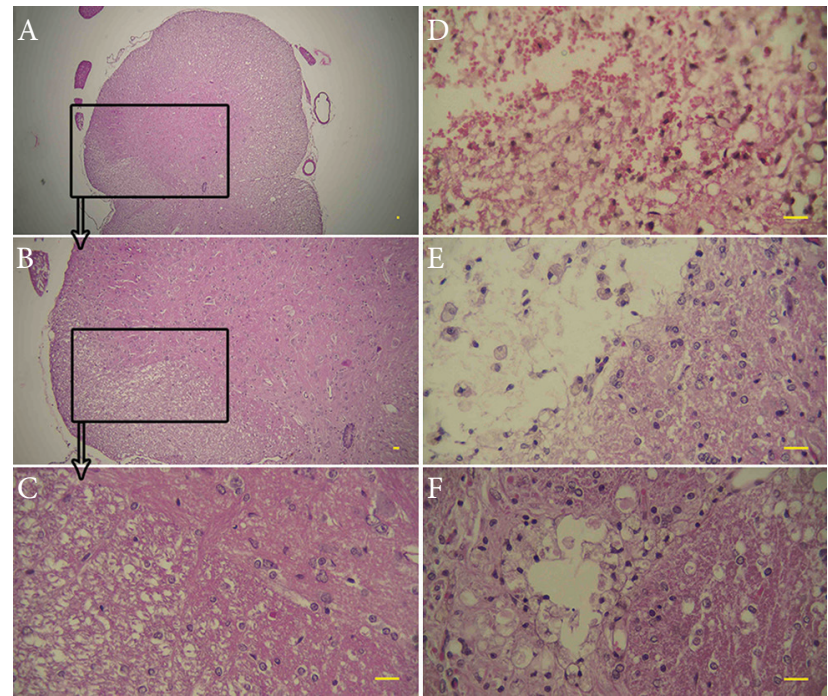

Fig. 2 Flaxseed reduced histological damage after SCI. Hematoxylin and eosin staining showed progressive destruction of the dorsal white matter and central grey matter in $\mathrm{SCl}$ rats compared with the control group. In the treatment group, the volume of induced cavity significantly reduced in comparison with the SCl group. A (4X), B (10X), C (40X): Control. D (40X): 3 days after SCI. E (40X): SCI. F (40X): Treatment. Scale bar $=100 \mu \mathrm{m}$.

\section{Discussion}

SCI is a cause of major neurological disability which is followed by great psychological disruption that includes both primary and secondary injuries. ${ }^{16}$ The primary injury of the spinal cord is followed by a long period of secondary injury. One of the most important factors involved in posttraumatic degeneration is the level of oxidative stress. ${ }^{5}$ Oxidative stress could lead to lipid peroxidation, DNA and protein oxidation and other disruptions. ${ }^{17,18}$ Oxidative and anti-oxidative parameters such as MDA, GPx, SOD and CAT have been studied in experimental SCI model. The increased level of MDA, SOD and decreased level of CAT, GPx have been reported in SCI rat model ${ }^{19}$ Liu et al. showed that oxidative stress level increased during first weeks after injury. ${ }^{20} \mathrm{MDA}$ is the end product of lipid peroxidation, and the content of MDA is an indicator of free radical levels. ${ }^{21}$ SOD with clearing $\mathrm{O}_{2}$ and preventing tissue injury by toxic oxygen-free radicals can be an indicator of antioxidant activity. ${ }^{22}$ Therefore, the level of SOD and MDA are important biomarkers of oxidative stress status. In this study, we showed that the mean level of SOD, GPx and CAT decreased in the SCI group, while the MDA content increased in comparison to the laminectomy group. Our results confirm that the stress oxidative level will increase as secondary bad consequences of SCI, which may lead to following neurons disruption. Up to now, researchers reported the influences of bilateral SCI on stress oxidative level, while in this study we represented that even unilateral SCI can disrupt the balance of stress oxidative condition. Therefore, reduction of oxidation stress level can be a key factor in the therapeutic schedule of SCI.

Flaxseed (Linum usitatissimum) is the seed from the flax plant, an annual herb, which is a rich source of plant omega-3 fatty acid a-linolenic acid, lignan phytoestrogen, and soluble fibres. ${ }^{10,11}$ There are some studies that have indicated the remarkable antioxidant ${ }^{12}$ and anti-inflammatory effects of flaxseed due to high levels of free radical scavenger properties and Omega-3 fatty acids, respectively. ${ }^{13}$ Lignan has been shown to provide neuroprotective and

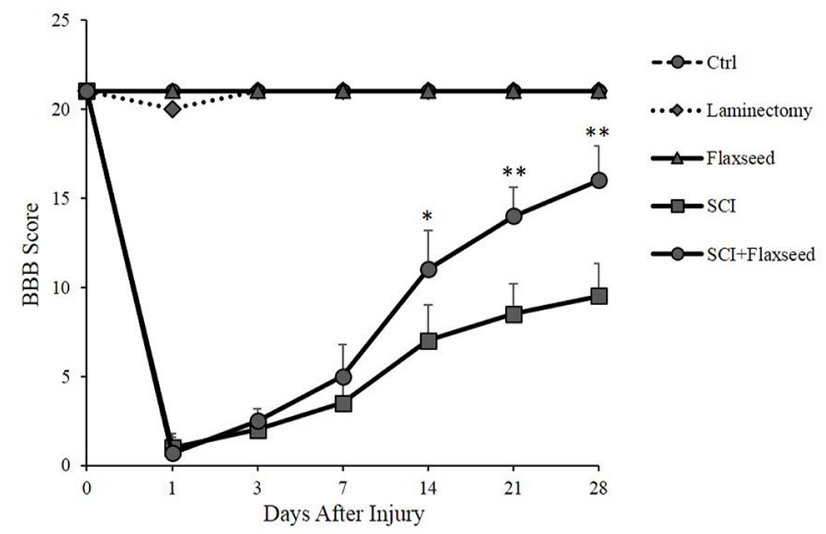

Fig. 3 Flaxseeds improve locomotor functional recovery of rats following $\mathrm{SCl}$. BBB test represented the significant increase in flaxseed-treated rats on days $14(P<0.05), 21$ and $28(P<001)$ after surgery compared with the $\mathrm{SCl}$ group. While, there are not any significant differences in $\mathrm{BBB}$ scores between the $\mathrm{SCl}$ and the treatment group on days 3 and 7 after $\mathrm{SCl}$. Data were presented as mean+SD. Ctrl: Control. ${ }^{*} P<0.05 ;{ }^{* *} P<0.001$. 
anti-oxidative effect against neurodegenerative diseases such as SCI. ${ }^{23}$ In 2006, King et al. reported that omega-3 fatty acid can improve SCI. ${ }^{24}$ In vitro study showed that flaxseed not only acts as a scavenger of hydroxyl radicals, but also inhibits the lipid peroxidation. Scientific evidences support the role of flaxseed in the prevention of some chronic diseases such as many types of cancer, diabetes, cardiovascular diseases and cerebrovascular stroke due to their biological active components. ${ }^{25-28}$ Moreover, it has been reported that flaxseed improves biochemical and oxidative stress parameters in patients with metabolic syndrome (MS).$^{29}$ In 2016, one research group reported that flaxseed oil (FSO) has a neuroprotective effect against $\mathrm{CCl} 4$-induced brain injury in gamma-irradiated rats. This effect is interrelated to the ability of FSO to scavenge the free radicals, enhances the antioxidant enzyme activity, increases glutathione contents and down-regulates the inflammatory responses. ${ }^{30}$

Based on previous studies, flaxseed has a significant therapeutic potential to decrease the level of oxidative stress. In the present study, for the first time to our knowledge, we assessed the effect of flaxseed in the unilateral SCI model. We found that 4 weeks after SCI induction GPx, SOD and CAT were markedly increased in the treatment group compared with control, whereas the MDA level was decreased. Therefore, we verified that flaxseed can suppress the oxidative stress in SCI.
In addition, our investigation demonstrated that flaxseed decreased tissue degeneration and the size of contusioninduced cavities in the treatment group, which resulted in improvement of motor activities. To evaluate the effect of flaxseed on locomotor function, animals were exposed to BBB test. Animals in the treatment group had significantly more recovery of motor function compared to SCI animals. It indicates that locomotor recovery after SCI in rats showed significant improvements with flaxseed treatment, as was confirmed by decreases in oxidative stress as a secondary injury.

In summary, our results showed that treatment with flaxseed diminished tissue degeneration and improved locomotor function, which can be explained by a reduction in the oxidative stress level after unilateral SCI. According to our results, flaxseed introduces as a new neuroprotective factor in SCI, and it needs to be more investigated in the future.

\section{Acknowledgments}

This work was supported by the Tehran University of Medical Sciences [grant number: 95-04-30-33618].

\section{Conflicts of Interest}

Authors have declared that no competing interests exist.

\section{References}

1. Pickett GE, Campos-Benitez M, Keller JL, Duggal N. Epidemiology of traumatic spinal cord injury in Canada. Spine. 2006:31:799-805.

2. Balentine JD. Pathology of experimental spinal cord trauma. I. The necrotic lesion as a function of vascular injury. Lab Invest. 1978;39: 236-253.

3. Cortez SC, McIntosh TK, Noble LJ. Experimental fluid percussion brain injury: vascular disruption and neuronal and glial alterations. Brain Res. 1989; 482:271-282.

4. Zhang HY, Wang ZG, Wu FZ, Kong XX, Yang J, Lin BB, et al. Regulation of autophagy and ubiquitinated protein accumulation by bFGF promotes functional recovery and neural protection in a rat model of spinal cord injury. Mol Neurobiol. 2013;48:452-464.

5. Chen HC, Hsu PW, Tzaan WC, Lee AW. Effects of the combined administration of vitamins $C$ and $E$ on the oxidative stress status and programmed cell death pathways after experimental spinal cord injury. Spinal Cord. 2014;52:24-28

6. Guéraud F, Atalay M, Bresgen N, Cipak A, Eckl PM, Huc L, et al. Chemistry and biochemistry of lipid peroxidation products. Free Radic Res. 2010;44: 1098-1124.

7. Nazıroğlu M. TRPM2 cation channels, oxidative stress and neurological diseases: where are we now?. Neurochem Res. 2011;36:355-366.

8. Somogyi A, Rosta K, Pusztai P, Tulassay Z, Nagy G. Antioxidant measurements. Physiol Meas. 2007;28:R41-55.

9. Rubilar M, Gutiérrez C, Verdugo M, Shene C, Sineiro J. Flaxseed as a source of functional ingredients. J Soil Sci Plant Nutr. 2010;10:373-377.

10. Oomah BD. Flaxseed as a functional food source. J Sci Food Agri. 2001:81:889-894.

11. Pengilly NL. 13 Traditional food and medicinal uses of flaxseed. Flax: the genus Linum. 2003:252.

12. Touré A, Xueming X. Flaxseed lignans: source, biosynthesis, metabolism, antioxidant activity, bio-active components, and health benefits. Comp Rev Food Sci Food Saf. 2010;9:261-269.

13. Calder PC. Polyunsaturated fatty acids, inflammation, and immunity. Lipids, 2001;36:1007-1024.

14. Winter R. Vitamin E: your protection against exercise fatigue, weakened immunity, heart disease, cancer, aging, diabetic damage, environmental toxins: Three Rivers Press. 2013

15. Basso DM, Beattie MS, Bresnahan JC. A sensitive and reliable locomotor rating scale for open field testing in rats. J Neurotrauma. $1995 ; 12: 1-21$.

16. Hong Z, Chen $H$, Hong H, Lin L, Wang Z. TSP-1 expression changes in diabetic rats with spinal cord injury. Neurol Res. 2009;31:878-882.

17. Dumont RJ, Okonkwo DO, Verma S, Hurlbert RJ, Boulos PT, Ellegala DB, et al. Acute spinal cord injury, part I: pathophysiologic mechanisms. Clin Neuropharmacol. 2001;24:254-264.

18. Fatima G, Sharma VP, Das SK, Mahdi AA. Oxidative stress and antioxidative parameters in patients with spinal cord injury: implications in the pathogenesis of disease. Spinal Cord. 2015:53:3-6.

19. Varija D, Kumar K, Reddy K, Reddy V. Prolonged constriction of sciatic nerve affecting oxidative stressors \& antioxidant enzymes in rat. Indian J Med Res. 2009;129:587-592.

20. Liu D, Liu J, Wen J. Elevation of hydrogen peroxide after spinal cord injury detected by using the Fenton reaction. Free Radic Biol Med. 1999;27:478-482.

21. Yue C, Chen J, Hou R, Liu J, Li X, Gao Z, et al. Effects of selenylation modification on antioxidative activities of schisandra chinensis polysaccharide. PLoS ONE. 2015;10:e0134363.

22. Carda AP, Marchi KC, Rizzi E, Mecawi AS, Antunes-Rodrigues J, Padovan $C M$, et al. Acute restraint stress induces endothelial dysfunction: role of vasoconstrictor prostanoids and oxidative stress. Stress. 2015;18:233-243.

23. Khalatbary AR. Natural polyphenols and spinal cord injury. Iran Biomed J. 2014;18:120-129

24. King VR, Huang WL, Dyall SC, Curran OE, Priestley JV, Michael-Titus AT. Omega-3 fatty acids improve recovery, whereas omega- 6 fatty acids worsen outcome, after spinal cord injury in the adult rat. J Neurosci. 2006;26: 4672-4680.

25. Adolphe JL, Whiting SJ, Juurlink BH, Thorpe LU, Alcorn J. Health effects with consumption of the flax lignan secoisolariciresinol diglucoside. Br J Nutr. 2010;103:929-938.

26. Kinniry P, Amrani Y, Vachani A, Solomides CC, Arguiri E, Workman A, et al. Dietary flaxseed supplementation ameliorates inflammation and oxidative tissue damage in experimental models of acute lung injury in mice. J Nutr. 2006;136:1545-1551. 
27. Lee JC, Bhora F, Sun J, Cheng G, Arguiri E, Solomides CC, et al. Dietary flaxseed enhances antioxidant defenses and is protective in a mouse model of lung ischemia-reperfusion injury. Am J Physiol Lung Cell Mol Physiol. 2008;294:L255-265.

28. Lee JC, Krochak R, Blouin A, Kanterakis S, Chatterjee S, Arguiri E, et al. Dietary flaxseed prevents radiation-induced oxidative lung damage, inflammation and fibrosis in a mouse model of thoracic radiation injury. Cancer Biol Ther. 2009:8:47-53.
29. Pilar BC, da Costa Güllich AA, Ströher DJ, Zuravski L, Mezzomo J, Coelho RP, et al. 28-days dietary supplementation with golden flaxseed improves biochemical and oxidative parameters in patients with metabolic syndrome. J Functional Foods. 2014;10:232-242.

30. Ismail AF, Salem AA, Eassawy MM. Modulation of gamma-irradiation and carbon tetrachloride induced oxidative stress in the brain of female rats by flaxseed oil. J Photochem Photobiol B. 2016; 161:91-99.

This work is licensed under a Creative Commons Attribution-NonCommercial 3.0 Unported License which allows users to read, copy, distribute and make derivative works for non-commercial purposes from the material, as long as the author of the original work is cited properly. 